CARPATHIAN J. MATH.

Volume 38 (2022), No. 1,

Pages $249-262$
Online version at https : //www . carpathian. cunbm. utcluj. ro/

Print Edition: ISSN 1584 - 2851; Online Edition: ISSN 1843 - 4401

DOI: https://doi.org/10.37193/CJM.2022.01.20

Dedicated to the memory of Academician Mitrofan M. Choban (1942-2021)

\title{
Halpern subgradient extragradient algorithm for solving quasimonotone variational inequality problems
}

\author{
PONGSAKORN YOtKaEW ${ }^{1}$, Habib UR REHMAN ${ }^{2}$, BANCHA PANYANAK ${ }^{3,4}$ and \\ NUTTAPOL PAKKARANANG ${ }^{5, \dagger}$
}

\begin{abstract}
In this paper, we study the numerical solution of the variational inequalities involving quasimonotone operators in infinite-dimensional Hilbert spaces. We prove that the iterative sequence generated by the proposed algorithm for the solution of quasimonotone variational inequalities converges strongly to a solution. The main advantage of the proposed iterative schemes is that it uses a monotone and non-monotone step size rule based on operator knowledge rather than its Lipschitz constant or some other line search method.
\end{abstract}

\section{INTRODUCTION}

Assume that $\mathcal{E}$ is a real Hilbert space and $\mathcal{K}$ be a nonempty closed convex subset of $\mathcal{E}$. Let $\mathcal{L}: \mathcal{E} \rightarrow \mathcal{E}$ be an operator. The problem (VIP) for $\mathcal{L}$ on $\mathcal{K}$ is defined as follows [17, 24]:

$$
\text { Find } x^{*} \in \mathcal{K} \text { such that }\left\langle\mathcal{L}\left(x^{*}\right), y-x^{*}\right\rangle \geq 0, \forall y \in \mathcal{K} \text {. }
$$

Our main concern here is to study the iterative methods that are used to approximate the solution of the variational inequality problem (shortly, VIP) involving quasimonotone operators in any real Hilbert space. In order to prove the strong convergence, it is considered that the following conditions have been satisfied:

$(\mathcal{L} 1)$ The solution set of a problem $(\mathrm{VIP})$ is denoted by $V I(\mathcal{K}, \mathcal{L})$ and it is nonempty;

$(\mathcal{L} 2)$ An operator $\mathcal{L}: \mathcal{E} \rightarrow \mathcal{E}$ is said to be quasimonotone if

$$
\left\langle\mathcal{L}\left(y_{1}\right), y_{2}-y_{1}\right\rangle>0 \Longrightarrow\left\langle\mathcal{L}\left(y_{2}\right), y_{1}-y_{2}\right\rangle \leq 0, \forall y_{1}, y_{2} \in \mathcal{K}
$$

$(\mathcal{L} 3)$ An operator $\mathcal{L}: \mathcal{E} \rightarrow \mathcal{E}$ is said to be Lipschitz continuous if there exists a constant $L>0$ such that

$$
\left\|\mathcal{L}\left(y_{1}\right)-\mathcal{L}\left(y_{2}\right)\right\| \leq L\left\|y_{1}-y_{2}\right\|, \forall y_{1}, y_{2} \in \mathcal{K}
$$

$(\mathcal{L} 4)$ An operator $\mathcal{L}: \mathcal{E} \rightarrow \mathcal{E}$ is said to be sequentially weakly continuous if $\left\{\mathcal{L}\left(x_{n}\right)\right\}$ converges weakly to $\mathcal{L}(x)$ for each sequence $\left\{x_{n}\right\}$ converges weakly to $x$.

It is well-established that the problem (VIP) is an important problem in the field of nonlinear analysis. It is an important mathematical model that unifies many crucial concepts in applied mathematics, such as a nonlinear system of equation, optimization conditions for problems with the optimization problems, the complementarity problems, the network equilibrium problems and finance (see for more details [8, 12, 13, 15, 16, 21, 25]). As

Received: 30.03.2021. In revised form: 09.08.2021. Accepted: 16.09.2021

2010 Mathematics Subject Classification. 47J25, 47H05, 47H10, 90C2, 47J25.

Key words and phrases. Subgradient extragradient method, Variational inequality problem, Strong convergence theorems, Quasimonotone mapping, Lipschitz continuity.

Corresponding author: ${ }^{\dagger}$ Nuttapol Pakkaranang; nuttapol.pak@pcru.ac.th 
a consequence, this concept has various applications in the field of engineering, mathematical programming, network economics, transport analysis, game theory and computer science.

The regularized and the projection methods are two prominent and general iterative schemes to approximate a solution to the variational inequalities. It is also noted that the first approach is most commonly used to solve variational inequalities accompanied by the class of monotone operators. The regularized subproblem in this method is strongly monotone and its unique solution exists more conveniently than the initial problem. In this paper, we look at some well-known projection methods that are well-known for their ease of numerical computation. The first well-known projection method is the gradient projection method that is used to solve variational inequalities. Moreover, several other projection methods have been established including the well-known extragradient method [18] the subgradient extragradient method [4, 5] and others in [6, 26, 20, 30, 11] and others in $[22,7,23,14,9,27,28,2,1,10]$. The above numerical techniques are used to examine the variational inequalities involving monotone, strongly monotone, or inverse monotone. The common feature of these methods is that fixed or variable step size rules are frequently used in constructing approximation solutions and establishing their convergence, depending on the Lipschitz constant of the involved operator. This can limit implementations because these parameters may be undefined or difficult to approximate in some situations.

The aim of this paper is to examine the quasimonotone variational inequalities in infinitedimensional Hilbert space and to verify that the iterative sequence proposed by the extragradient algorithm for solving quasimonotone variational inequalities converges strongly to a solution. The proposed subgradient extragradient algorithm uses both the monotone and the new non-monotone variable step size rule.

The paper is arranged in the following manner. In Sect. 2, some preliminary results were presented. Sect. 3 gives two new algorithms and their convergence analysis. Finally, Sect. 4 gives some numerical results to explain the practical efficiency of the proposed methods.

\section{PRELIMINARIES}

For all $x, y \in \mathcal{E}$, we have

$$
\|x+y\|^{2}=\|x\|^{2}+2\langle x, y\rangle+\|y\|^{2} .
$$

A metric projection $P_{\mathcal{K}}\left(y_{1}\right)$ of $y_{1} \in \mathcal{E}$ is defined by

$$
P_{\mathcal{K}}\left(y_{1}\right)=\arg \min \left\{\left\|y_{1}-y_{2}\right\|: y_{2} \in \mathcal{K}\right\} .
$$

Lemma 2.1. [3] For all $y_{1}, y_{2} \in \mathcal{E}$ and $\ell \in \mathbb{R}$. Then

(i) $\left\|\ell y_{1}+(1-\ell) y_{2}\right\|^{2}=\ell\left\|y_{1}\right\|^{2}+(1-\ell)\left\|y_{2}\right\|^{2}-\ell(1-\ell)\left\|y_{1}-y_{2}\right\|^{2}$.

(ii) $\left\|y_{1}+y_{2}\right\|^{2} \leq\left\|y_{1}\right\|^{2}+2\left\langle y_{2}, y_{1}+y_{2}\right\rangle$.

Lemma 2.2. [29] Let $\left\{p_{n}\right\} \subset[0,+\infty)$ be a sequence such that

$$
p_{n+1} \leq\left(1-q_{n}\right) p_{n}+q_{n} r_{n}, \forall n \in \mathbb{N} .
$$

Moreover, two sequences $\left\{q_{n}\right\} \subset(0,1)$ and $\left\{r_{n}\right\} \subset \mathbb{R}$ satisfying the following conditions:

$$
\lim _{n \rightarrow+\infty} q_{n}=0, \sum_{n=1}^{+\infty} q_{n}=+\infty \text { and } \limsup _{n \rightarrow+\infty} r_{n} \leq 0 \text {. }
$$

Then, $\lim _{n \rightarrow+\infty} p_{n}=0$. 
Lemma 2.3. [19] Let $\left\{p_{n}\right\}$ be a sequence and there exists a subsequence $\left\{n_{i}\right\}$ of $\{n\}$ such that

$$
p_{n_{i}}<p_{n_{i+1}}, \forall i \in \mathbb{N} .
$$

Then, there exists a nondecreasing sequence $m_{k} \subset \mathbb{N}$ such that $m_{k} \rightarrow+\infty$ as $k \rightarrow+\infty$, and satisfying the following inequality for $k \in \mathbb{N}$ :

$$
p_{m_{k}} \leq p_{m_{k+1}} \text { and } p_{k} \leq p_{m_{k+1}} .
$$

Indeed, $m_{k}=\max \left\{j \leq k: p_{j} \leq p_{j+1}\right\}$.

\section{MAIN RESUlTS}

In this section, we propose an initial method to solve quasimonotone variational inequalities in real Hilbert spaces and prove a strong convergence result for the proposed method. The first method involves the monotonic self-adaptive step rule to make the method independent of the Lipschitz constant. The first method is written as follows:

Algorithm 1 (Monotonic Explicit Halpern-Type Subgradient Extragradient Method)

Step 0: Let $x_{1} \in \mathcal{K}, \rho_{1}>0, \mu \in(0,1)$ and $\left\{\gamma_{n}\right\} \subset(0,1)$ meet the following conditions:

$$
\lim _{n \rightarrow+\infty} \gamma_{n}=0 \text { and } \sum_{n=1}^{+\infty} \gamma_{n}=+\infty \text {. }
$$

Step 1: Compute $y_{n}=P_{\mathcal{K}}\left(x_{n}-\rho_{n} \mathcal{L}\left(x_{n}\right)\right)$. If $x_{n}=y_{n}$, STOP. Otherwise, go to Step 2.

Step 2: Compute $z_{n}=P_{\mathcal{E}_{n}}\left(x_{n}-\rho_{n} \mathcal{L}\left(y_{n}\right)\right)$ where

$$
\mathcal{E}_{n}=\left\{z \in \mathcal{E}:\left\langle x_{n}-\rho_{n} \mathcal{L}\left(x_{n}\right)-y_{n}, z-y_{n}\right\rangle \leq 0\right\}
$$

Step 3: Compute $x_{n+1}=\gamma_{n} x_{1}+\left(1-\gamma_{n}\right) z_{n}$.

Step 4: Compute

$$
\rho_{n+1}=\left\{\begin{array}{lc}
\min \left\{\rho_{n}, \frac{\mu\left\|x_{n}-y_{n}\right\|^{2}+\mu\left\|z_{n}-y_{n}\right\|^{2}}{2\left[\left\langle\mathcal{L}\left(x_{n}\right)-\mathcal{L}\left(y_{n}\right), z_{n}-y_{n}\right\rangle\right]}\right\} & \text { if } \quad\left\langle\mathcal{L}\left(x_{n}\right)-\mathcal{L}\left(y_{n}\right), z_{n}-y_{n}\right\rangle>0 \\
\rho_{n}, & \text { otherwise }
\end{array}\right.
$$

Set $n:=n+1$ and go back to Step 1 .

Lemma 3.4. A sequence $\left\{\rho_{n}\right\}$ generated by (3.1) is monotonically decreasing and convergent.

Proof. Due to the Lipschitz continuity of a mapping $\mathcal{L}$ there exists a fixed number $L>0$. Suppose that $\left\langle\mathcal{L}\left(x_{n}\right)-\mathcal{L}\left(y_{n}\right), z_{n}-y_{n}\right\rangle>0$ such that

$$
\begin{aligned}
\frac{\mu\left(\left\|x_{n}-y_{n}\right\|^{2}+\left\|z_{n}-y_{n}\right\|^{2}\right)}{2\left\langle\mathcal{L}\left(x_{n}\right)-\mathcal{L}\left(y_{n}\right), z_{n}-y_{n}\right\rangle} & \geq \frac{2 \mu\left\|x_{n}-y_{n}\right\|\left\|z_{n}-y_{n}\right\|}{2\left\|\mathcal{L}\left(x_{n}\right)-\mathcal{L}\left(y_{n}\right)\right\|\left\|z_{n}-y_{n}\right\|} \\
& \geq \frac{2 \mu\left\|x_{n}-y_{n}\right\|\left\|z_{n}-y_{n}\right\|}{2 L\left\|x_{n}-y_{n}\right\|\left\|z_{n}-y_{n}\right\|} \geq \frac{\mu}{L} .
\end{aligned}
$$

We can easily determine that the sequence $\left\{\rho_{n}\right\}$ is bounded and monotonically decreasing. Hence, sequence $\left\{\rho_{n}\right\}$ is convergent to some $\rho>0$.

Lemma 3.5. Let $\mathcal{L}: \mathcal{E} \rightarrow \mathcal{E}$ be an operator satisfies the conditions $(\mathcal{L} 1)-(\mathcal{L} 4)$. For $x^{*} \in$ $V I(\mathcal{K}, \mathcal{L}) \neq \emptyset$, we have

$$
\left\|z_{n}-x^{*}\right\|^{2} \leq\left\|x_{n}-x^{*}\right\|^{2}-\left(1-\frac{\mu \rho_{n}}{\rho_{n+1}}\right)\left\|x_{n}-y_{n}\right\|^{2}-\left(1-\frac{\mu \rho_{n}}{\rho_{n+1}}\right)\left\|z_{n}-y_{n}\right\|^{2} .
$$


Proof. Now consider that

$$
\begin{aligned}
\left\|z_{n}-x^{*}\right\|^{2}= & \left\|P_{\mathcal{E}_{n}}\left[x_{n}-\rho_{n} \mathcal{L}\left(y_{n}\right)\right]-x^{*}\right\|^{2} \\
= & \left\|P_{\mathcal{E}_{n}}\left[x_{n}-\rho_{n} \mathcal{L}\left(y_{n}\right)\right]+\left[x_{n}-\rho_{n} \mathcal{L}\left(y_{n}\right)\right]-\left[x_{n}-\rho_{n} \mathcal{L}\left(y_{n}\right)\right]-x^{*}\right\|^{2} \\
= & \left\|\left[x_{n}-\rho_{n} \mathcal{L}\left(y_{n}\right)\right]-x^{*}\right\|^{2}+\left\|P_{\mathcal{E}_{n}}\left[x_{n}-\rho_{n} \mathcal{L}\left(y_{n}\right)\right]-\left[x_{n}-\rho_{n} \mathcal{L}\left(y_{n}\right)\right]\right\|^{2} \\
& +2\left\langle P_{\mathcal{E}_{n}}\left[x_{n}-\rho_{n} \mathcal{L}\left(y_{n}\right)\right]-\left[x_{n}-\rho_{n} \mathcal{L}\left(y_{n}\right)\right],\left[x_{n}-\rho_{n} \mathcal{L}\left(y_{n}\right)\right]-x^{*}\right\rangle .
\end{aligned}
$$

By using $x^{*} \in V I(\mathcal{K}, \mathcal{L}) \subset \mathcal{K} \subset \mathcal{E}_{n}$, we get

$$
\begin{aligned}
& \left\|P_{\mathcal{E}_{n}}\left[x_{n}-\rho_{n} \mathcal{L}\left(y_{n}\right)\right]-\left[x_{n}-\rho_{n} \mathcal{L}\left(y_{n}\right)\right]\right\|^{2} \\
& \quad+\left\langle P_{\mathcal{E}_{n}}\left[x_{n}-\rho_{n} \mathcal{L}\left(y_{n}\right)\right]-\left[x_{n}-\rho_{n} \mathcal{L}\left(y_{n}\right)\right],\left[x_{n}-\rho_{n} \mathcal{L}\left(y_{n}\right)\right]-x^{*}\right\rangle \\
& =\left\langle\left[x_{n}-\rho_{n} \mathcal{L}\left(y_{n}\right)\right]-P_{\mathcal{E}_{n}}\left[x_{n}-\rho_{n} \mathcal{L}\left(y_{n}\right)\right], x^{*}-P_{\mathcal{E}_{n}}\left[x_{n}-\rho_{n} \mathcal{L}\left(y_{n}\right)\right]\right\rangle \leq 0 .
\end{aligned}
$$

Thus, above expression implies that

$$
\begin{aligned}
& \left\langle P_{\mathcal{E}_{n}}\left[x_{n}-\rho_{n} \mathcal{L}\left(y_{n}\right)\right]-\left[x_{n}-\rho_{n} \mathcal{L}\left(y_{n}\right)\right],\left[x_{n}-\rho_{n} \mathcal{L}\left(y_{n}\right)\right]-x^{*}\right\rangle \\
& \leq-\left\|P_{\mathcal{E}_{n}}\left[x_{n}-\rho_{n} \mathcal{L}\left(y_{n}\right)\right]-\left[x_{n}-\rho_{n} \mathcal{L}\left(y_{n}\right)\right]\right\|^{2} .
\end{aligned}
$$

From expressions (3.3) and (3.5), we obtain

$$
\begin{aligned}
\left\|z_{n}-x^{*}\right\|^{2} & \leq\left\|x_{n}-\rho_{n} \mathcal{L}\left(y_{n}\right)-x^{*}\right\|^{2}-\left\|P_{\mathcal{E}_{n}}\left[x_{n}-\rho_{n} \mathcal{L}\left(y_{n}\right)\right]-\left[x_{n}-\rho_{n} \mathcal{L}\left(y_{n}\right)\right]\right\|^{2} \\
& \leq\left\|x_{n}-x^{*}\right\|^{2}-\left\|x_{n}-z_{n}\right\|^{2}+2 \rho_{n}\left\langle\mathcal{L}\left(y_{n}\right), x^{*}-z_{n}\right\rangle
\end{aligned}
$$

Since $x^{*}$ is the solution of problem (VIP), we have

$$
\left\langle\mathcal{L}\left(x^{*}\right), y-x^{*}\right\rangle \geq 0, \text { for all } y \in \mathcal{K} .
$$

Due to the mapping $\mathcal{L}$ on $\mathcal{K}$, we obtain

$$
\left\langle\mathcal{L}(y), y-x^{*}\right\rangle \geq 0, \text { for all } y \in \mathcal{K} .
$$

By substituting $y=y_{n} \in \mathcal{K}$, we get

$$
\left\langle\mathcal{L}\left(y_{n}\right), y_{n}-x^{*}\right\rangle \geq 0 .
$$

Thus, we have

$$
\left\langle\mathcal{L}\left(y_{n}\right), x^{*}-z_{n}\right\rangle=\left\langle\mathcal{L}\left(y_{n}\right), x^{*}-y_{n}\right\rangle+\left\langle\mathcal{L}\left(y_{n}\right), y_{n}-z_{n}\right\rangle \leq\left\langle\mathcal{L}\left(y_{n}\right), y_{n}-z_{n}\right\rangle .
$$

Combining expressions (3.6) and (3.7), we obtain

$$
\begin{aligned}
\left\|z_{n}-x^{*}\right\|^{2} & \leq\left\|x_{n}-x^{*}\right\|^{2}-\left\|x_{n}-z_{n}\right\|^{2}+2 \rho_{n}\left\langle\mathcal{L}\left(y_{n}\right), y_{n}-z_{n}\right\rangle \\
& \leq\left\|x_{n}-x^{*}\right\|^{2}-\left\|x_{n}-y_{n}+y_{n}-z_{n}\right\|^{2}+2 \rho_{n}\left\langle\mathcal{L}\left(y_{n}\right), y_{n}-z_{n}\right\rangle \\
& \leq\left\|x_{n}-x^{*}\right\|^{2}-\left\|x_{n}-y_{n}\right\|^{2}-\left\|y_{n}-z_{n}\right\|^{2}+2\left\langle x_{n}-\rho_{n} \mathcal{L}\left(y_{n}\right)-y_{n}, z_{n}-y_{n}\right\rangle .
\end{aligned}
$$

Note that $z_{n}=P_{\mathcal{E}_{n}}\left[x_{n}-\rho_{n} \mathcal{L}\left(y_{n}\right)\right]$ and by the definition of $\rho_{n+1}$, we have

$$
\begin{aligned}
& 2\left\langle x_{n}-\rho_{n} \mathcal{L}\left(y_{n}\right)-y_{n}, z_{n}-y_{n}\right\rangle \\
& =2\left\langle x_{n}-\rho_{n} \mathcal{L}\left(x_{n}\right)-y_{n}, z_{n}-y_{n}\right\rangle+2 \rho_{n}\left\langle\mathcal{L}\left(x_{n}\right)-\mathcal{L}\left(y_{n}\right), z_{n}-y_{n}\right\rangle \\
& \leq \frac{\rho_{n}}{\rho_{n+1}} 2 \rho_{n+1}\left\langle\mathcal{L}\left(x_{n}\right)-\mathcal{L}\left(y_{n}\right), z_{n}-y_{n}\right\rangle \\
& \leq \frac{\mu \rho_{n}}{\rho_{n+1}}\left\|x_{n}-y_{n}\right\|^{2}+\frac{\mu \rho_{n}}{\rho_{n+1}}\left\|z_{n}-y_{n}\right\|^{2} .
\end{aligned}
$$


Combining expressions (3.8) and (3.9), we obtain

$$
\begin{aligned}
& \left\|z_{n}-x^{*}\right\|^{2} \\
& \leq\left\|x_{n}-x^{*}\right\|^{2}-\left\|x_{n}-y_{n}\right\|^{2}-\left\|y_{n}-z_{n}\right\|^{2}+\frac{\rho_{n}}{\rho_{n+1}}\left[\mu\left\|x_{n}-y_{n}\right\|^{2}+\mu\left\|z_{n}-y_{n}\right\|^{2}\right] \\
& \leq\left\|x_{n}-x^{*}\right\|^{2}-\left(1-\frac{\mu \rho_{n}}{\rho_{n+1}}\right)\left\|x_{n}-y_{n}\right\|^{2}-\left(1-\frac{\mu \rho_{n}}{\rho_{n+1}}\right)\left\|z_{n}-y_{n}\right\|^{2} .
\end{aligned}
$$

Lemma 3.6. Let $\mathcal{L}: \mathcal{E} \rightarrow \mathcal{E}$ be an operator satisfying the conditions $(\mathcal{L} 1)-(\mathcal{L} 4)$. If there exists a subsequence $\left\{x_{n_{k}}\right\}$ weakly convergent to $\hat{x}$ and $\lim _{k \rightarrow \infty}\left\|x_{n_{k}}-y_{n_{k}}\right\|=0$. Then, $\hat{x}$ is the solution of the problem (VIP).

Proof. Since $\left\{x_{n_{k}}\right\}$ weakly convergent to $\hat{x}$ and due to $\lim _{k \rightarrow \infty}\left\|x_{n_{k}}-y_{n_{k}}\right\|=0$, sequence $\left\{y_{n_{k}}\right\}$ also weakly convergent to $\hat{x}$. Next, we need to prove that $\hat{x} \in V I(\mathcal{K}, \mathcal{L})$. Indeed, we have

that is equivalent to

$$
y_{n_{k}}=P_{\mathcal{K}}\left[x_{n_{k}}-\rho_{n_{k}} \mathcal{L}\left(x_{n_{k}}\right)\right]
$$

$$
\left\langle x_{n_{k}}-\rho_{n_{k}} \mathcal{L}\left(x_{n_{k}}\right)-y_{n_{k}}, y-y_{n_{k}}\right\rangle \leq 0, \forall y \in \mathcal{K} .
$$

The inequality mentioned above implies that

$$
\left\langle x_{n_{k}}-y_{n_{k}}, y-y_{n_{k}}\right\rangle \leq \rho_{n_{k}}\left\langle\mathcal{L}\left(x_{n_{k}}\right), y-y_{n_{k}}\right\rangle, \forall y \in \mathcal{K}
$$

Thus, we obtain

$$
\frac{1}{\rho_{n_{k}}}\left\langle x_{n_{k}}-y_{n_{k}}, y-y_{n_{k}}\right\rangle+\left\langle\mathcal{L}\left(x_{n_{k}}\right), y_{n_{k}}-x_{n_{k}}\right\rangle \leq\left\langle\mathcal{L}\left(x_{n_{k}}\right), y-x_{n_{k}}\right\rangle, \forall y \in \mathcal{K} .
$$

Since $\min \left\{\frac{\mu}{L}, \rho_{1}\right\} \leq \rho \leq \rho_{1}$ and $\left\{x_{n_{k}}\right\}$ is a bounded sequence. By the use of $\lim _{k \rightarrow \infty} \| x_{n_{k}}-$ $y_{n_{k}} \|=0$ and $k \rightarrow \infty$ in expression (3.13), we obtain

$$
\liminf _{k \rightarrow \infty}\left\langle\mathcal{L}\left(x_{n_{k}}\right), y-x_{n_{k}}\right\rangle \geq 0, \forall y \in \mathcal{K} \text {. }
$$

Moreover, we have

$$
\begin{aligned}
& \left\langle\mathcal{L}\left(y_{n_{k}}\right), y-y_{n_{k}}\right\rangle \\
& =\left\langle\mathcal{L}\left(y_{n_{k}}\right)-\mathcal{L}\left(x_{n_{k}}\right), y-x_{n_{k}}\right\rangle+\left\langle\mathcal{L}\left(x_{n_{k}}\right), y-x_{n_{k}}\right\rangle+\left\langle\mathcal{L}\left(y_{n_{k}}\right), x_{n_{k}}-y_{n_{k}}\right\rangle .
\end{aligned}
$$

Since $\lim _{k \rightarrow \infty}\left\|x_{n_{k}}-y_{n_{k}}\right\|=0$ and $\mathcal{L}$ is $L$-Lipschitz continuity on $\mathcal{E}$ implies that

$$
\lim _{k \rightarrow \infty}\left\|\mathcal{L}\left(x_{n_{k}}\right)-\mathcal{L}\left(y_{n_{k}}\right)\right\|=0,
$$

which together with expressions (3.15) and (3.16), we obtain

$$
\liminf _{k \rightarrow \infty}\left\langle\mathcal{L}\left(y_{n_{k}}\right), y-y_{n_{k}}\right\rangle \geq 0, \forall y \in \mathcal{K} .
$$

To prove further, let us take a positive sequence $\left\{\epsilon_{k}\right\}$ that is convergent to zero and decreasing. For each $\left\{\epsilon_{k}\right\}$ we denote by $m_{k}$ the smallest positive integer such that

$$
\left\langle\mathcal{L}\left(x_{n_{i}}\right), y-x_{n_{i}}\right\rangle+\epsilon_{k}>0, \forall i \geq m_{k}
$$

where the existence of $m_{k}$ follows from expression (3.17). Since $\left\{\epsilon_{k}\right\}$ is decreasing and it is easy to see that the sequence $m_{k}$ is increasing.

Case I: If there is a subsequence $\left\{x_{n_{m_{k_{j}}}}\right\}$ of $\left\{x_{n_{m_{k}}}\right\}$ such that $\mathcal{L}\left(x_{n_{m_{k_{j}}}}\right)=0(\forall j)$. Let $j \rightarrow \infty$, we obtain

$$
\langle\mathcal{L}(\hat{x}), y-\hat{x}\rangle=\lim _{j \rightarrow \infty}\left\langle\mathcal{L}\left(x_{n_{m_{k_{j}}}}\right), y-\hat{x}\right\rangle=0 .
$$

Thus, $\hat{x} \in \mathcal{K}$ and imply that $\hat{x} \in V I(\mathcal{K}, \mathcal{L})$. 
Case II: If there exists $N_{0} \in \mathbb{N}$ such that for all $n_{m_{k}} \geq N_{0}, \mathcal{L}\left(x_{n_{m_{k}}}\right) \neq 0$. Consider that

$$
\Upsilon_{n_{m_{k}}}=\frac{\mathcal{L}\left(x_{n_{m_{k}}}\right)}{\left\|\mathcal{L}\left(x_{n_{m_{k}}}\right)\right\|^{2}}, \forall n_{m_{k}} \geq N_{0}
$$

Due to the above definition, we obtain

$$
\left\langle\mathcal{L}\left(x_{n_{m_{k}}}\right), \Upsilon_{n_{m_{k}}}\right\rangle=1, \forall n_{m_{k}} \geq N_{0}
$$

Moreover, expressions (3.18) and (3.21) for all $n_{m_{k}} \geq N_{0}$, we have

$$
\left\langle\mathcal{L}\left(x_{n_{m_{k}}}\right), y+\epsilon_{k} \Upsilon_{n_{m_{k}}}-x_{n_{m_{k}}}\right\rangle>0 .
$$

Since $\mathcal{L}$ is quasimonotone, then

$$
\left\langle\mathcal{L}\left(y+\epsilon_{k} \Upsilon_{n_{m_{k}}}\right), y+\epsilon_{k} \Upsilon_{n_{m_{k}}}-x_{n_{m_{k}}}\right\rangle>0 .
$$

For all $n_{m_{k}} \geq N_{0}$, we have

$$
\left\langle\mathcal{L}(y), y-x_{n_{m_{k}}}\right\rangle \geq\left\langle\mathcal{L}(y)-\mathcal{L}\left(y+\epsilon_{k} \Upsilon_{n_{m_{k}}}\right), y+\epsilon_{k} \Upsilon_{n_{m_{k}}}-x_{n_{m_{k}}}\right\rangle-\epsilon_{k}\left\langle\mathcal{L}(y), \Upsilon_{n_{m_{k}}}\right\rangle
$$

Due to $\left\{x_{n_{k}}\right\}$ weakly converges to $\hat{x} \in \mathcal{K}$ through $\mathcal{L}$ is sequentially weakly continuous on the set $\mathcal{K}$, we get $\left\{\mathcal{L}\left(x_{n_{k}}\right)\right\}$ weakly converges to $\mathcal{L}(\hat{x})$. Suppose that $\mathcal{L}(\hat{x}) \neq 0$, we have

$$
\|\mathcal{L}(\hat{x})\| \leq \liminf _{k \rightarrow \infty}\left\|\mathcal{L}\left(x_{n_{k}}\right)\right\| .
$$

Since $\left\{x_{n_{m_{k}}}\right\} \subset\left\{x_{n_{k}}\right\}$ and $\lim _{k \rightarrow \infty} \epsilon_{k}=0$, we have

$$
0 \leq \lim _{k \rightarrow \infty}\left\|\epsilon_{k} \Upsilon_{n_{m_{k}}}\right\|=\lim _{k \rightarrow \infty} \frac{\epsilon_{k}}{\left\|\mathcal{L}\left(x_{n_{m_{k}}}\right)\right\|} \leq \frac{0}{\|\mathcal{L}(\hat{x})\|}=0 .
$$

Next, consider $k \rightarrow \infty$ in (3.24), we obtain

$$
\langle\mathcal{L}(y), y-\hat{x}\rangle \geq 0, \forall y \in \mathcal{K} .
$$

Let $x \in \mathcal{K}$ be arbitrary element and for $0<\lambda \leq 1$, let

$$
\hat{x}_{\lambda}=\lambda x+(1-\lambda) \hat{x} \text {. }
$$

Then $\hat{x}_{\lambda} \in \mathcal{K}$ and from (3.27) we have

$$
\lambda\left\langle\mathcal{T}\left(\hat{x}_{\lambda}\right), x-\hat{x}\right\rangle \geq 0 .
$$

Thus, we have

$$
\left\langle\mathcal{T}\left(\hat{x}_{\lambda}\right), x-\hat{x}\right\rangle \geq 0
$$

Let $\lambda \rightarrow 0$. Then $\hat{x}_{\lambda} \rightarrow \hat{x}$ along a line segment. By the continuity of an operator, $\mathcal{T}\left(\hat{x}_{\lambda}\right)$ converges to $\mathcal{T}(\hat{x})$ as $\lambda \rightarrow 0$. It follows from (3.30) that

$$
\langle\mathcal{T}(\hat{x}), x-\hat{x}\rangle \geq 0 \text {. }
$$

Therefore, $\hat{x}$ is a solution of the problem (VIP).

Theorem 3.1. Let $\mathcal{L}: \mathcal{E} \rightarrow \mathcal{E}$ be an operator satisfies the conditions $(\mathcal{L} 1)-(\mathcal{L} 4)$. Then, the sequence $\left\{x_{n}\right\}$ generated by the Algorithm 1 converges strongly to a solution $x^{*} \in V I(\mathcal{K}, \mathcal{L})$.

Proof. From Lemma 3.5, we have

$$
\left\|z_{n}-x^{*}\right\|^{2} \leq\left\|x_{n}-x^{*}\right\|^{2}, \forall n \geq n_{1} .
$$

Since $\rho_{n} \rightarrow \rho$, thus there exists a fixed number $\epsilon \in(0,1-\mu)$ such that

$$
\lim _{n \rightarrow \infty}\left(1-\frac{\mu \rho_{n}}{\rho_{n+1}}\right)=1-\mu>\epsilon>0 .
$$


Thus, there exists a finite number $n_{1} \in \mathbb{N}$ such that

$$
\left(1-\frac{\mu \rho_{n}}{\rho_{n+1}}\right)>\epsilon>0, \forall n \geq n_{1} .
$$

By the use of definition of $\left\{x_{n+1}\right\}$ we obtain

$$
\begin{aligned}
\left\|x_{n+1}-x^{*}\right\| & =\left\|\alpha_{n} x_{1}+\left(1-\alpha_{n}\right) z_{n}-x^{*}\right\| \\
& =\left\|\alpha_{n}\left[x_{1}-x^{*}\right]+\left(1-\alpha_{n}\right)\left[z_{n}-x^{*}\right]\right\| \\
& \leq \alpha_{n}\left\|x_{1}-x^{*}\right\|+\left(1-\alpha_{n}\right)\left\|z_{n}-x^{*}\right\| .
\end{aligned}
$$

Combining expressions (3.33) and (3.34), we obtain

$$
\begin{aligned}
\left\|x_{n+1}-x^{*}\right\| & \leq \alpha_{n}\left\|x_{1}-x^{*}\right\|+\left(1-\alpha_{n}\right)\left\|x_{n}-x^{*}\right\| . \\
& \leq \max \left\{\left\|x_{1}-x^{*}\right\|,\left\|x_{n}-x^{*}\right\|\right\} \\
& \leq \max \left\{\left\|x_{1}-x^{*}\right\|,\left\|x_{n_{1}}-x^{*}\right\|\right\} .
\end{aligned}
$$

Thus, we conclude that $\left\{x_{n}\right\}$ is a bounded sequence. By using Lemma 2.1 (i), we have

$$
\begin{aligned}
\left\|x_{n+1}-x^{*}\right\|^{2}= & \left\|\alpha_{n} x_{1}+\left(1-\alpha_{n}\right) z_{n}-x^{*}\right\|^{2} \\
= & \left\|\alpha_{n}\left[x_{1}-x^{*}\right]+\left(1-\alpha_{n}\right)\left[z_{n}-x^{*}\right]\right\|^{2} \\
= & \alpha_{n}\left\|x_{1}-x^{*}\right\|^{2}+\left(1-\alpha_{n}\right)\left\|z_{n}-x^{*}\right\|^{2}-\alpha_{n}\left(1-\alpha_{n}\right)\left\|x_{1}-z_{n}\right\|^{2} \\
\leq & \alpha_{n}\left\|x_{1}-x^{*}\right\|^{2}+\left(1-\alpha_{n}\right)\left[\left\|x_{n}-x^{*}\right\|^{2}-\left(1-\frac{\mu \rho_{n}}{\rho_{n+1}}\right)\left\|x_{n}-y_{n}\right\|^{2}\right. \\
& \left.-\left(1-\frac{\mu \rho_{n}}{\rho_{n+1}}\right)\left\|z_{n}-y_{n}\right\|^{2}\right]-\alpha_{n}\left(1-\alpha_{n}\right)\left\|x_{1}-z_{n}\right\|^{2} \\
\leq & \alpha_{n}\left\|x_{1}-x^{*}\right\|^{2}+\left\|x_{n}-x^{*}\right\|^{2} \\
& -\left(1-\alpha_{n}\right)\left(1-\frac{\mu \rho_{n}}{\rho_{n+1}}\right)\left\|x_{n}-y_{n}\right\|^{2}-\left(1-\alpha_{n}\right)\left(1-\frac{\mu \rho_{n}}{\rho_{n+1}}\right)\left\|z_{n}-y_{n}\right\|^{2} .
\end{aligned}
$$

The above relation implies that

$$
\begin{aligned}
& \left(1-\alpha_{n}\right)\left(1-\frac{\mu \rho_{n}}{\rho_{n+1}}\right)\left\|x_{n}-y_{n}\right\|^{2}+\left(1-\alpha_{n}\right)\left(1-\frac{\mu \rho_{n}}{\rho_{n+1}}\right)\left\|z_{n}-y_{n}\right\|^{2} \\
& \leq \alpha_{n}\left\|x_{1}-x^{*}\right\|^{2}+\left\|x_{n}-x^{*}\right\|^{2}-\left\|x_{n+1}-x^{*}\right\|^{2} .
\end{aligned}
$$

From Lemma 2.1, we have

$$
\begin{aligned}
\left\|x_{n+1}-x^{*}\right\|^{2} & =\left\|\alpha_{n} x_{1}+\left(1-\alpha_{n}\right) z_{n}-x^{*}\right\|^{2} \\
& =\left\|\alpha_{n}\left[x_{1}-x^{*}\right]+\left(1-\alpha_{n}\right)\left[z_{n}-x^{*}\right]\right\|^{2} \\
& \leq\left(1-\alpha_{n}\right)^{2}\left\|z_{n}-x^{*}\right\|^{2}+2 \alpha_{n}\left\langle x_{1}-x^{*},\left(1-\alpha_{n}\right)\left[z_{n}-x^{*}\right]+\alpha_{n}\left[x_{1}-x^{*}\right]\right\rangle \\
& =\left(1-\alpha_{n}\right)^{2}\left\|z_{n}-x^{*}\right\|^{2}+2 \alpha_{n}\left\langle x_{1}-x^{*}, x_{n+1}-x^{*}\right\rangle \\
& \leq\left(1-\alpha_{n}\right)\left\|x_{n}-x^{*}\right\|^{2}+2 \alpha_{n}\left\langle x_{1}-x^{*}, x_{n+1}-x^{*}\right\rangle .
\end{aligned}
$$

Case 1: Assume that there exists a fixed number $n_{2} \in \mathbb{N}$ such that

$$
\left\|x_{n+1}-x^{*}\right\| \leq\left\|x_{n}-x^{*}\right\|, \forall n \geq n_{2} .
$$


Thus, the above expression implies that $\lim _{n \rightarrow \infty}\left\|x_{n}-x^{*}\right\|$ exists and let $\lim _{n \rightarrow \infty} \| x_{n}-$ $x^{*} \|=l$. From expression (3.37), we have

$$
\begin{aligned}
& \left(1-\alpha_{n}\right)\left(1-\frac{\mu \rho_{n}}{\rho_{n+1}}\right)\left\|x_{n}-y_{n}\right\|^{2}+\left(1-\alpha_{n}\right)\left(1-\frac{\mu \rho_{n}}{\rho_{n+1}}\right)\left\|z_{n}-y_{n}\right\|^{2} \\
& \leq \alpha_{n}\left\|x_{1}-x^{*}\right\|^{2}+\left\|x_{n}-x^{*}\right\|^{2}-\left\|x_{n+1}-x^{*}\right\|^{2} .
\end{aligned}
$$

The existence of $\lim _{n \rightarrow \infty}\left\|x_{n}-x^{*}\right\|=l$, and $\alpha_{n} \rightarrow 0$ we can deduce that

$$
\lim _{n \rightarrow \infty}\left\|x_{n}-y_{n}\right\|=\lim _{n \rightarrow \infty}\left\|z_{n}-y_{n}\right\|=0 .
$$

It follows that

$$
\lim _{n \rightarrow \infty}\left\|x_{n}-z_{n}\right\| \leq \lim _{n \rightarrow \infty}\left\|x_{n}-y_{n}\right\|+\lim _{n \rightarrow \infty}\left\|y_{n}-z_{n}\right\|=0
$$

Furthermore, we obtain

$$
\begin{aligned}
\left\|x_{n+1}-x_{n}\right\| & =\left\|\alpha_{n} x_{1}+\left(1-\alpha_{n}\right) z_{n}-x_{n}\right\| \\
& =\left\|\alpha_{n}\left[x_{1}-x_{n}\right]+\left(1-\alpha_{n}\right)\left[z_{n}-x_{n}\right]\right\| \\
& \leq \alpha_{n}\left\|x_{1}-x_{n}\right\|+\left(1-\alpha_{n}\right)\left\|z_{n}-x_{n}\right\| .
\end{aligned}
$$

It follows that

$$
\lim _{n \rightarrow \infty}\left\|x_{n+1}-x_{n}\right\|=0
$$

Since $\left\{x_{n}\right\}$ is bounded sequence and there exists a subsequence $\left\{x_{n_{k}}\right\}$ that converges weakly to some $\hat{x} \in \mathcal{E}$. By using Lemma 3.6, we have

$$
\begin{aligned}
& \limsup _{n \rightarrow \infty}\left\langle x_{1}-x^{*}, x_{n}-x^{*}\right\rangle \\
& =\limsup _{k \rightarrow \infty}\left\langle x_{1}-x^{*}, x_{n_{k}}-x^{*}\right\rangle=\left\langle x_{1}-x^{*}, \hat{x}-x^{*}\right\rangle \leq 0 .
\end{aligned}
$$

Since $x^{*}=P_{V I(\mathcal{K}, \mathcal{L})}\left(x_{1}\right)$. Thus, we have

$$
\left\langle x_{1}-x^{*}, y-x^{*}\right\rangle \leq 0, \forall y \in V I(\mathcal{K}, \mathcal{L}) .
$$

Combining expressions (3.45) and (3.46), we obtain

$$
\begin{aligned}
& \limsup _{n \rightarrow \infty}\left\langle x_{1}-x^{*}, x_{n+1}-x^{*}\right\rangle \\
& \leq \limsup _{n \rightarrow \infty}\left\langle x_{1}-x^{*}, x_{n+1}-x_{n}\right\rangle+\limsup _{n \rightarrow \infty}\left\langle x_{1}-x^{*}, x_{n}-x^{*}\right\rangle \leq 0 .
\end{aligned}
$$

Case 2: Assume that there exists a subsequence $\left\{n_{i}\right\}$ of $\{n\}$ such that

$$
\left\|x_{n_{i}}-x^{*}\right\| \leq\left\|x_{n_{i+1}}-x^{*}\right\|, \forall i \in \mathbb{N} .
$$

Thus, by Lemma 2.3 there exists a sequence $\left\{m_{k}\right\} \subset \mathbb{N}$ as $\left\{m_{k}\right\} \rightarrow \infty$, such that

$$
\left\|x_{m_{k}}-x^{*}\right\| \leq\left\|x_{m_{k+1}}-x^{*}\right\| \quad \text { and } \quad\left\|x_{k}-x^{*}\right\| \leq\left\|x_{m_{k+1}}-x^{*}\right\|, \text { for all } k \in \mathbb{N} \text {. }
$$

Similar to Case 1 and expression (3.37) provides that

$$
\begin{aligned}
& \left(1-\alpha_{m_{k}}\right)\left(1-\frac{\mu \rho_{m_{k}}}{\rho_{m_{k}+1}}\right)\left\|x_{m_{k}}-y_{m_{k}}\right\|^{2}+\left(1-\alpha_{m_{k}}\right)\left(1-\frac{\mu \rho_{m_{k}}}{\rho_{m_{k}+1}}\right)\left\|z_{m_{k}}-y_{m_{k}}\right\|^{2} \\
& \leq \alpha_{m_{k}}\left\|x_{1}-x^{*}\right\|^{2}+\left\|x_{m_{k}}-x^{*}\right\|^{2}-\left\|x_{m_{k}+1}-x^{*}\right\|^{2} .
\end{aligned}
$$

Due to $\alpha_{m_{k}} \rightarrow 0$, we deduce the following results:

$$
\lim _{n \rightarrow \infty}\left\|x_{m_{k}}-y_{m_{k}}\right\|=\lim _{n \rightarrow \infty}\left\|z_{m_{k}}-y_{m_{k}}\right\|=0 .
$$


Next, we can obtain

$$
\begin{aligned}
\left\|x_{m_{k}+1}-x_{m_{k}}\right\| & =\left\|\alpha_{m_{k}} x_{1}+\left(1-\alpha_{m_{k}}\right) z_{m_{k}}-x_{m_{k}}\right\| \\
& =\left\|\alpha_{m_{k}}\left[x_{1}-x_{m_{k}}\right]+\left(1-\alpha_{m_{k}}\right)\left[z_{m_{k}}-x_{m_{k}}\right]\right\| \\
& \leq \alpha_{m_{k}}\left\|x_{1}-x_{m_{k}}\right\|+\left(1-\alpha_{m_{k}}\right)\left\|z_{m_{k}}-x_{m_{k}}\right\| \longrightarrow 0 .
\end{aligned}
$$

We use the same argument as in Case 1, which is as follows:

$$
\limsup _{k \rightarrow \infty}\left\langle x_{1}-x^{*}, x_{m_{k}+1}-x^{*}\right\rangle \leq 0 .
$$

Now, using expressions (3.38), we have

$$
\begin{aligned}
\left\|x_{m_{k}+1}-x^{*}\right\|^{2} & \leq\left(1-\alpha_{m_{k}}\right)\left\|x_{m_{k}}-x^{*}\right\|^{2}+2 \alpha_{m_{k}}\left\langle x_{1}-x^{*}, x_{m_{k}+1}-x^{*}\right\rangle \\
& \leq\left(1-\alpha_{m_{k}}\right)\left\|x_{m_{k}+1}-x^{*}\right\|^{2}+2 \alpha_{m_{k}}\left\langle x_{1}-x^{*}, x_{m_{k}+1}-x^{*}\right\rangle
\end{aligned}
$$

It continues from that

$$
\left\|x_{m_{k}+1}-x^{*}\right\|^{2} \leq 2\left\langle x_{1}-x^{*}, x_{m_{k}+1}-x^{*}\right\rangle .
$$

Thus, expressions (3.47) and (3.54) implies that

$$
\left\|x_{m_{k}+1}-x^{*}\right\|^{2} \rightarrow 0 \text {, as } k \rightarrow \infty .
$$

It implies that

$$
\lim _{n \rightarrow \infty}\left\|x_{k}-x^{*}\right\|^{2} \leq \lim _{n \rightarrow \infty}\left\|x_{m_{k}+1}-x^{*}\right\|^{2} \leq 0 .
$$

Consequently, $x_{n} \rightarrow x^{*}$. This completes the proof of the theorem.

Now, we propose a second variant of the first method to solve quasimonotone variational inequalities in real Hilbert spaces and prove a strong convergence result for the proposed method. The second method involves a non-monotonic self adaptive step rule to make the method independent of the Lipschitz constant. The second method is written as follows:

Algorithm 2 (Non-Monotonic Explicit Halpern-Type Subgradient Extragradient Method)

Step 0: Let $x_{1} \in \mathcal{K}, \rho_{1}>0, \mu \in(0,1)$ and sequence $\left\{\varphi_{n}\right\}$ satisfying $\sum_{n=1}^{+\infty} \varphi_{n}<+\infty$. Moreover, $\left\{\gamma_{n}\right\} \subset(0,1)$ satisfying the following conditions:

$$
\lim _{n \rightarrow+\infty} \gamma_{n}=0 \text { and } \sum_{n=1}^{+\infty} \gamma_{n}=+\infty
$$

Step 1: Compute $y_{n}=P_{\mathcal{K}}\left(x_{n}-\rho_{n} \mathcal{L}\left(x_{n}\right)\right)$. If $x_{n}=y_{n}$, STOP. Otherwise, go to Step 2.

Step 2: Compute $z_{n}=P_{\mathcal{E}_{n}}\left(x_{n}-\rho_{n} \mathcal{L}\left(y_{n}\right)\right)$ where

$$
\mathcal{E}_{n}=\left\{z \in \mathcal{E}:\left\langle x_{n}-\rho_{n} \mathcal{L}\left(x_{n}\right)-y_{n}, z-y_{n}\right\rangle \leq 0\right\} .
$$

Step 3: Compute $x_{n+1}=\gamma_{n} x_{1}+\left(1-\gamma_{n}\right) z_{n}$.

Step 4: Compute

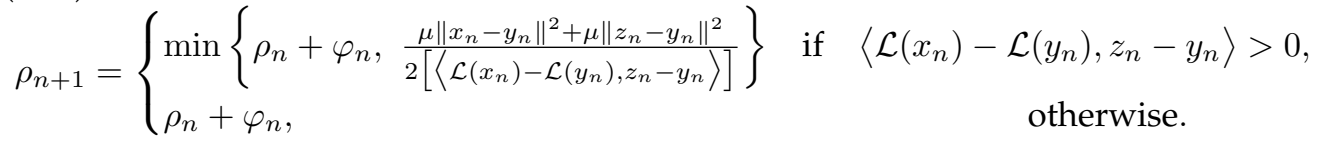

Set $n:=n+1$ and go back to Step 1 . 
Lemma 3.7. A sequence $\left\{\rho_{n}\right\}$ generated by (3.57) is convergent to $\rho$ and satisfying the following inequality

$$
\min \left\{\frac{\mu}{L}, \rho_{1}\right\} \leq \rho_{n} \leq \rho_{1}+P \text { where } P=\sum_{n=1}^{+\infty} \varphi_{n} .
$$

Proof. Due to the Lipschitz continuity of a mapping $\mathcal{L}$ there exists a fixed number $L>0$. Consider that $\left\langle\mathcal{L}\left(x_{n}\right)-\mathcal{L}\left(y_{n}\right), z_{n}-y_{n}\right\rangle>0$, implies that

$$
\begin{aligned}
\frac{\mu\left(\left\|x_{n}-y_{n}\right\|^{2}+\left\|z_{n}-y_{n}\right\|^{2}\right)}{2\left\langle\mathcal{L}\left(x_{n}\right)-\mathcal{L}\left(y_{n}\right), z_{n}-y_{n}\right\rangle} & \geq \frac{2 \mu\left\|x_{n}-y_{n}\right\|\left\|z_{n}-y_{n}\right\|}{2\left\|\mathcal{L}\left(x_{n}\right)-\mathcal{L}\left(y_{n}\right)\right\|\left\|z_{n}-y_{n}\right\|} \\
& \geq \frac{2 \mu\left\|x_{n}-y_{n}\right\|\left\|z_{n}-y_{n}\right\|}{2 L\left\|x_{n}-y_{n}\right\|\left\|z_{n}-y_{n}\right\|} \geq \frac{\mu}{L} .
\end{aligned}
$$

By using mathematical induction on the definition of $\rho_{n+1}$, we have

$$
\min \left\{\frac{\mu}{L}, \rho_{1}\right\} \leq \rho_{n} \leq \rho_{1}+P .
$$

Let $\left[\rho_{n+1}-\rho_{n}\right]^{+}=\max \left\{0, \rho_{n+1}-\rho_{n}\right\}$ and $\left[\rho_{n+1}-\rho_{n}\right]^{-}=\max \left\{0,-\left(\rho_{n+1}-\rho_{n}\right)\right\}$. From the definition of $\left\{\rho_{n}\right\}$, we have

$$
\sum_{n=1}^{+\infty}\left(\rho_{n+1}-\rho_{n}\right)^{+}=\sum_{n=1}^{+\infty} \max \left\{0, \rho_{n+1}-\rho_{n}\right\} \leq P<+\infty .
$$

That is, the series $\sum_{n=1}^{+\infty}\left(\rho_{n+1}-\rho_{n}\right)^{+}$is convergent. Next, we need to prove the convergence of $\sum_{n=1}^{+\infty}\left(\rho_{n+1}-\rho_{n}\right)^{-}$. Let $\sum_{n=1}^{+\infty}\left(\rho_{n+1}-\rho_{n}\right)^{-}=+\infty$. Due to the reason that $\rho_{n+1}-\rho_{n}=$ $\left(\rho_{n+1}-\rho_{n}\right)^{+}-\left(\rho_{n+1}-\rho_{n}\right)^{-}$. Thus, we have

$$
\rho_{k+1}-\rho_{1}=\sum_{n=0}^{k}\left(\rho_{n+1}-\rho_{n}\right)=\sum_{n=0}^{k}\left(\rho_{n+1}-\rho_{n}\right)^{+}-\sum_{n=0}^{k}\left(\rho_{n+1}-\rho_{n}\right)^{-} .
$$

By allowing $k \rightarrow+\infty$ in (3.60), we have $\rho_{k} \rightarrow-\infty$ as $k \rightarrow \infty$. This is a contradiction. Due to the convergence of the series $\sum_{n=0}^{k}\left(\rho_{n+1}-\rho_{n}\right)^{+}$and $\sum_{n=0}^{k}\left(\rho_{n+1}-\rho_{n}\right)^{-}$taking $k \rightarrow+\infty$ in (3.60), we obtain $\lim _{n \rightarrow \infty} \rho_{n}=\rho$. This completes the proof.

Theorem 3.2. Let a mapping $\mathcal{L}: \mathcal{E} \rightarrow \mathcal{E}$ satisfies the condition $(\mathcal{L} 1)-(\mathcal{L} 4)$. Then, the sequence $\left\{x_{n}\right\}$ generated by the Algorithm 2 converges strongly to a solution of $\operatorname{VI}(\mathcal{K}, \mathcal{L})$.

Proof. The proof is the same as of Theorem 3.1.

\section{Numerical IlLUSTRATIONS}

The computational results of the proposed schemes are described in this section and study how variations in control parameters affect the numerical effectiveness of the proposed algorithms. All computations are done in MATLAB R2018b and run on HP i 5 Core(TM)i5-6200 8.00 GB (7.78 GB usable) RAM laptop.

Example 4.1. Let $\mathcal{E}=l_{2}$ be a real Hilbert space with sequences of real numbers satisfying the following condition

$$
\left\|x_{1}\right\|^{2}+\left\|x_{2}\right\|^{2}+\cdots+\left\|x_{n}\right\|^{2}+\cdots<+\infty
$$


Assume that operator $\mathcal{L}: \mathcal{K} \rightarrow \mathcal{K}$ is defined by

$$
G(x)=(5-\|x\|) x, \forall x \in \mathcal{E}
$$

where $\mathcal{K}=\{x \in \mathcal{E}:\|x\| \leq 3\}$. It is easy to see that $\mathcal{L}$ is weakly sequentially continuous on $\mathcal{E}$ and $V I(\mathcal{K}, \mathcal{L})=\{0\}$. For any $x, y \in \mathcal{E}$, we have

$$
\begin{aligned}
\|\mathcal{L}(x)-\mathcal{L}(y)\| & =\|(5-\|x\|) x-(5-\|y\|) y\| \\
& =\|5(x-y)-\| x\|(x-y)-(\|x\|-\|y\|) y\| \\
& \leq 5\|x-y\|+\|x\|\|x-y\|+\|\| x\|-\| y\|\|\|y\| \\
& \leq 5\|x-y\|+3\|x-y\|+3\|x-y\| \\
& \leq 11\|x-y\| .
\end{aligned}
$$

Hence $\mathcal{L}$ is $L$-Lipschitz continuous with $L=11$. For any $x, y \in \mathcal{E}$ and let $\langle\mathcal{L}(x), y-x\rangle>0$ such that

$$
(5-\|x\|)\langle x, y-x\rangle>0 .
$$

Since $\|x\| \leq 3$ implies that

$$
\langle x, y-x\rangle>0
$$

Thus, we have

$$
\begin{aligned}
\langle\mathcal{L}(y), y-x\rangle & =(5-\|y\|)\langle y, y-x\rangle \\
& \geq(5-\|y\|)\langle y, y-x\rangle-(5-\|y\|)\langle x, y-x\rangle \\
& \geq 2\|x-y\|^{2} \geq 0 .
\end{aligned}
$$

Thus, we shown that $\mathcal{L}$ is quasimonotone on $\mathcal{K}$. Let $x=\left(\frac{5}{2}, 0,0, \cdots, 0, \cdots\right)$ and $y=(3,0,0, \cdots, 0, \cdots)$, we have

$$
\langle\mathcal{L}(x)-\mathcal{L}(y), x-y\rangle=(2.5-3)^{3}<0 .
$$

A projection on the set $C$ is computed explicitly as follows:

$$
P_{C}(x)= \begin{cases}x & \text { if } \quad\|x\| \leq 3 \\ \frac{3 x}{\|x\|}, & \text { otherwise. }\end{cases}
$$

Numerical results are shown in Figures 1 and 2 and Table 1. The iterative control parameters are taken in the following manner: (i) Algorithm $1: \rho_{1}=0.20 ; \mu=0.55 ; \gamma_{n}=\frac{1}{(n+2)} ; D_{n}=$ $\left\|x_{n}-y_{n}\right\|$; (ii) Algorithm $2: \rho_{1}=0.20 ; \mu=0.55 ; \gamma_{n}=\frac{1}{(n+2)} ; \varphi_{n}=\frac{100}{(n+1)} ; D_{n}=\left\|x_{n}-y_{n}\right\|$.

TABLE 1. Numerical results values for Example 4.1.

\begin{tabular}{lllll}
\hline & \multicolumn{3}{c}{ Number of Iterations } & \multicolumn{2}{c}{ Execution Time in Seconds } \\
\hline$x_{1}$ & Algorithm 1 & Algorithm 2 & Algorithm 1 & Algorithm 2 \\
\hline$\left(1,1, \cdots, 1_{5000}, 0,0, \cdots\right)$ & 120 & 75 & 7.47764030000000 & 4.79321210000000 \\
$(1,2, \cdots, 5000,0,0, \cdots)$ & 220 & 72 & 14.3395908000000 & 6.83192160000000 \\
\hline
\end{tabular}



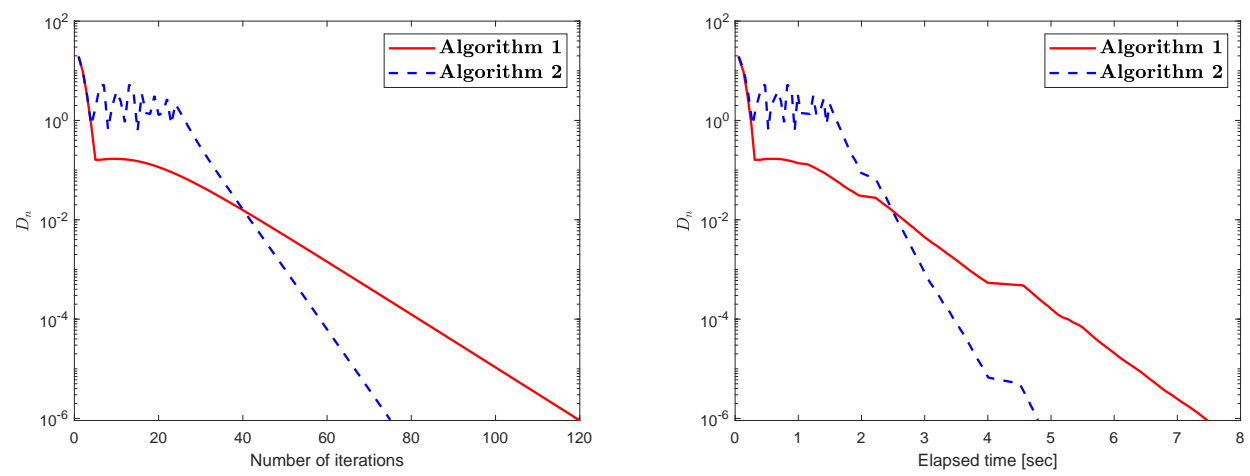

FIGURE 1. Numerical illustration of Algorithm 1 and Algorithm 2 by using $x_{1}=\left(1,1, \cdots, 1_{5000}, 0,0, \cdots\right)$.
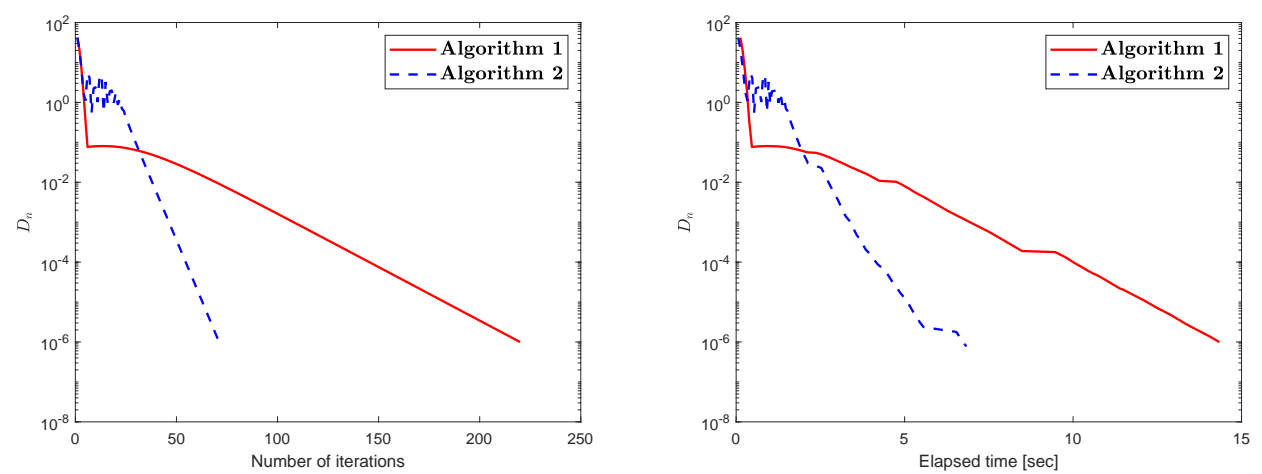

FIGURE 2. Numerical illustration of Algorithm 1 and Algorithm 2 by using $x_{1}=(1,2, \cdots, 5000,0,0, \cdots)$.

\section{CONCLUSION}

The main idea of this paper is to study quasimonotone variational inequality problems in infinite-dimensional Hilbert spaces and to prove that the iterative sequence generated by the Halpern subgradient extragradient algorithm is convergent strongly to a solution. Acknowledgement. The first author was supported by National Research Council of Thailand and Khon Kaen University 2017 (Grant on. 600052). The third author was support by Chiang Mai University. The fourth author would like to thank Phetchabun Rajabhat University.

\section{REFERENCES}

[1] Abubakar, J.; Kumam, P.; Ur Rehman, H. Self-adaptive inertial subgradient extragradient scheme for pseudomonotone variational inequality problem. Int. J. Nonlinear Sci. Numer. Simul. 2021, DOI:10.1515/ijnsns2020-0033 (Accepted)

[2] Abubakar, J.; Kumam, P.; Ur Rehman, H.; Ibrahim, A. H. Inertial iterative schemes with variable step sizes for variational inequality problem involving pseudomonotone operator. Mathematics 8 (2020), Article ID 609, 25 pp.

[3] Bauschke, H. H.; Combettes, P. L. Convex analysis and monotone operator theory in Hilbert spaces. Springer, New York, 2011 
[4] Censor, Y.; Gibali, A.; Reich, S. The subgradient extragradient method for solving variational inequalities in Hilbert space. J. Optim. Theory Appl. 163 (2014), 399-412

[5] Censor, Y.; Gibali, A.; Reich, S. Strong convergence of subgradient extragradient methods for the variational inequality problem in Hilbert space. Optim. Methods Softw. 26 (2011), 827-845

[6] Censor, Y.; Gibali, A.; Reich, S. Extensions of Korpelevich's extragradient method for the variational inequality problem in Euclidean space. Optimization, 61 (2012), 1119-1132

[7] Cholamjiak, P.; Thong, D. V. and Cho, Y. J. A novel inertial projection and contraction method for solving pseudomonotone variational inequality problems. Acta Appl. Math. 167 (2020), 217-245

[8] Elliott, C. M. Variational and quasivariational inequalities applications to free-boundary probLems (Claudio Baiocchi and António Capelo). SIAM Rev., 29 (1987), 314-315

[9] Garodia, C.; Uddin, I. A new iterative method for solving split feasibility problem. J. Appl. Anal. Comput. 10 (2020), 986-1004

[10] Hammad, H. A.; Ur Rehman, H.; la Sen, M. D. Advanced algorithms and common solutions to variational inequalities. Symmetry 12 (2020), Article ID 1198, 15 pp.

[11] Iusem, A. N.; Svaiter, B. F. A variant of Korpelevich's method for variational inequalities with a new search strategy. Optimization 42 (1997), 309-321

[12] Kassay, G.; Kolumbán, J.; Páles, Z. On nash stationary points. Publ. Math. Debrecen 54 (1999), 267-279

[13] Kassay, G., Kolumbán, J.; Páles, Z. Factorization of Minty and Stampacchia variational inequality systems. European J. Oper. Res. 143 (2002), 377-389

[14] Khatoon, S.; Cholamjiak, W.; Uddin, I. A modified proximal point algorithm involving nearly asymptotically quasi-nonexpansive mappings. J. Inequal. Appl. 2021 (2021), Article ID 83, 20 pp.

[15] Kinderlehrer, D.; Stampacchia, G. An introduction to variational inequalities and their applications. Society for Industrial and Applied Mathematics (SIAM), Philadelphia, 2000

[16] Konnov, I. Equilibrium models and variational inequalities. Elsevier B. V., Amsterdam, (2007)

[17] Konnov, I. V. On systems of variational inequalities. Izv. Vyssh. Uchebn. Zaved. Mat. 12 (1997), 79-88

[18] Korpelevich, G. The extragradient method for finding saddle points and other problems. Matekon 12 (1977), $35-49$

[19] Maingé, P. E. Strong convergence of projected subgradient methods for nonsmooth and nonstrictly convex minimization. Set-Valued Anal. 16 (2008), 899-912

[20] Moudafi, A. Viscosity approximation methods for fixed-points problems. J. Math. Anal. Appl. 241 (2000), $46-55$

[21] Nagurney, A. Network economics: a variational inequality approach, Kluwer Academic Publishers Group, Dordrecht, 1999

[22] Reich, S.; Thong, D. V.; Cholamjiak, P.; Luong, L. V. Inertial projection-type methods for solving pseudomonotone variational inequality problems in Hilbert space. Numer. Algorithms 2021, DOI:10.1007/s11075-020-01058-6

[23] Shehu, Y.; Cholamjiak, P. Iterative method with inertial for variational inequalities in Hilbert spaces. Calcolo 56 (2019), Article ID 4, 21 pp.

[24] Stampacchia, G., Formes bilinéaires coercitives sur les ensembles convexes, Académie des Sciences de Paris, 258 (1964), 4413-4416

[25] Takahashi, W. Introduction to nonlinear and convex analysis, Yokohama Publishers, Yokohama, 2009

[26] Tseng, P. A modified forward-backward splitting method for maximal monotone mappings. SIAM J. Control Optim. 38 (2000), 431-446

[27] Wairojjana, N.; Pakkaranang, N.; Ur Rehman, H.; Pholasa, N.; Khanpanuk, T. Strong convergence of extragradient-type method to solve pseudomonotone variational inequalities problems. Axioms 9 (2020), Article ID 115, 16 pp.

[28] Wairojjana, N.; Younis, M.; Ur Rehman, H.; Pakkaranang, N.; Pholasa, N., Modified viscosity subgradient extragradient-like algorithms for solving monotone variational inequalities problems. Axioms 9 (2020), Article ID 118, 19 pp.

[29] Xu, H. K. Another control condition in an iterative method for nonexpansive mappings. Bull. Austral. Math. Soc. 65 (2002), 109-113

[30] Zhang, L.; Fang, C.; Chen, S. An inertial subgradient-type method for solving single-valued variational inequalities and fixed point problems. Numer. Algorithms 79 (2018), 941-956

\footnotetext{
${ }^{1}$ DEPARTMENT OF MATHEMATICS

FACULTY OF SCIENCE

KHON KAEN UNIVERSITY

KHON KAEN 40002, THAILAND

Email address: pongyo@kku.ac.th
} 
2 Department of Mathematics

KING MONGKUT'S UNIVERSITY OF TECHNOLOGY THONBURI (KMUTT)

BANGKOK 10140, THAILAND

Email address: habib.rehman@mail.kmutt.ac.th

${ }^{3}$ Research Center in Mathematics And Applied Mathematics

DEPARTMENT OF MATHEMATICS

FACULTY OF SCIENCE, CHIANG MAI UNIVERSITY

CHIANG MAI 50200, THAILAND

${ }^{4}$ Data SCIENCE RESEARCH CENTER

DEPARTMENT OF MATHEMATICS

FACULTY OF SCIENCE

CHIANG MAI UNIVERSITY

CHIANG MAI 50200, THAILAND

Email address: bancha.p@cmu.ac.th

${ }^{5}$ Department of MATHEMATics

FACUlty of SCIENCE AND TECHNOLOGY

Phetchabun RajabHat University

Phetchabun 67000, Thailand.

Email address: nuttapol.pakepcru.ac.th 